\title{
The Bernese peri-acetabular osteotomy through a modified approach. A technical note
}

\author{
Paul Whittingham-Jones • Nirav Kirit Patel • \\ Aresh Hashemi-Nejad
}

Received: 20 August 2012/Accepted: 8 February 2013/Published online: 3 March 2013

(C) EPOS 2013

\begin{abstract}
Introduction A modification of the technique for performing the Bernese periacetabular osteotomy is described. Materials and methods A medial approach to the Ischium and a subtle re-orientation of the pubic osteotomy are detailed.

Conclusion Surgical morbidity is likely decreased with a concurrent cosmetic advantage without compromise to surgical correction or patient safety.
\end{abstract}

Keywords Peri-acetabular osteotomy $\cdot$ Hip $\cdot$ Approach

The peri-acetabular osteotomy represents the current state of evolution of a series of procedures which have attempted to re-orientate the dysplastic acetabulum. Useful in the mature skeleton, Ganz has popularised the technique, building on the previous triple innominate osteotomies of Tönnis. As the technique has progressed in the hands of different surgeons, various modifications have been described. We present a surgical technique designed to reduce the surgical morbidity of the procedure without compromise of the achievable bony correction or patient safety. The modifications described are a medial approach to the ischium and a subtle alteration of the orientation of the pubic osteotomy.

P. Whittingham-Jones $(\bowtie) \cdot$ N. Kirit Patel · A. Hashemi-Nejad Royal National Orthopaedic Hospital (RNOH), Stanmore,

Middlesex HA7 4LP, UK

e-mail: mrpaulwj@gmail.com

N. Kirit Patel

e-mail: niravpatel@doctors.org.uk

A. Hashemi-Nejad

e-mail: Aresh.Nejad@rnoh.nhs.uk

\section{Technique}

The patient is positioned supine on a radiolucent table. Standard fluoroscopic images are obtained to ensure appropriate positioning. In practice, this means confirming that it is possible to achieve an antero-posterior projection, an iliac-oblique projection, an obturator oblique projection and a false profile projection. The affected limb is draped free to the groin and up to the costal margin. The first osteotomy is the ischial cut. Ganz et al. [1] have described their approach to the joint capsule and infra-cotyloid groove via an extended Smith-Petersen approach. Ganz uses this interval to access the area below the acetabulum prior to the ischial osteotomy. This is a long incision, which is prone to unsightly scars and a high incidence of lateral femoral cutaneous nerve palsy. This is the site of the principal modification of the technique. A medial approach to the ischium is performed (Fig. 1). Via a $3-\mathrm{cm}$ incision perpendicular to the line of the adductor tendons, just distal to their origin, the adductor longus is identified. The plane posterior to the adductor longus is developed, over the adductor brevis, with identification of the obturator vessels and the anterior branch of the obturator nerve. The inferior margin of the hip capsule is followed with blunt dissection as far as the ischium. The transverse acetabular ligament is palpable. Overlying the bone is the obturator externus, which must be swept off. Two Hohmann retractors are placed around the bone, one inside the obturator foramen, the other lateral, which will protect the sciatic nerve, once it has exited the sciatic notch (Figs. 2 and 3). Their placement is confirmed via fluoroscopy. Next, a straight osteotome is placed at the infracotyloid groove, which is beneath the transverse acetabular ligament and extra-capsular. This osteotome is passed $1 \mathrm{~cm}$ through the lateral cortex towards the laterally placed Hohmann retractor. The 


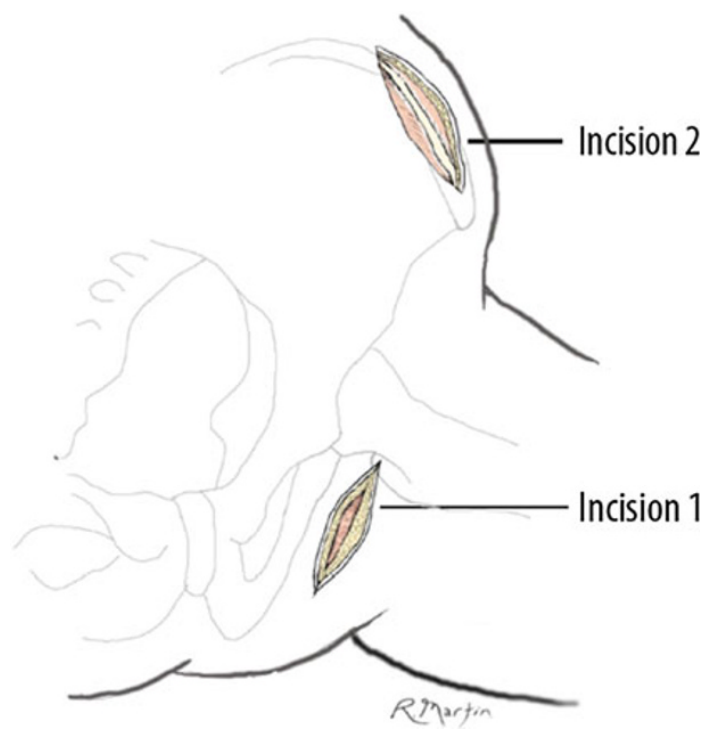

Fig. 1 Illustration of the two skin incisions

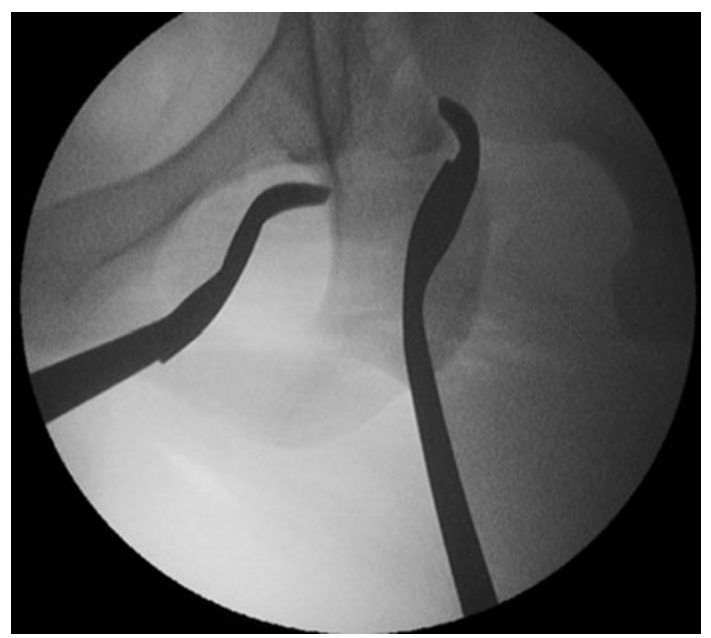

Fig. 2 Hohmann retractors around the ischium. The lateral retractor protects the sciatic nerve and, in combination with the medial retractor, the infracotyloid groove is well visualised

sciatic nerve is in danger here. The medial cortex is then breached with the same osteotome. The Ganz osteotome is then applied and directed up the posterior column, again under image guidance with an iliac-oblique projection (Fig. 4). Due to the orientation possible with this medial approach, the Ganz osteotome is able to advance to a level around or just past the ischial spine. This, in turn, facilitates the later osteotomy. Once this osteotomy has passed cephalad to a satisfactory distance, the osteotome is removed and a pack applied into the wound.

Attention then turns to the remaining osteotomies. A second incision is then made parallel and caudal to the iliac wing. The external oblique aponeurosis is elevated, so tissue is left for repair prior to exposure of the outer table of the ilium, which allows the insertion of a retractor for soft tissue protection. The abductors are preserved. Subperiosteal exposure of the area between the anterior superior iliac spine and the anterior inferior iliac spine is developed. The exposure is then developed down the inner table of the ilium, including elevation of the tough periosteum. An assistant to aid in the exposure of the pubis flexes the knee and hip, and the superior pubic ramus is exposed. A Hohmann retractor is placed medial to the palpable eminence (Fig. 5). Following meticulous periosteal release, a curved osteotomy is made of the pubis. The periosteal release here is of paramount importance. The periosteum encountered here is very tough and circumferential around the pubis, making it difficult to clear but, if not addressed, will prevent the necessary distraction of the pubic osteotomy (Fig. 6). The fluoroscopic image is now via the obturator oblique projection. The curved nature of this cut is essential, as it facilitates the later angled correction through this osteotomy. Once performed, it is important to check that the cut is complete via fluoroscopy and also that the osteotomy is mobile via palpation of the surrounding periosteal sleeve, which represents a potential block to subsequent correction. Any persistent periosteal sleeve is divided with cautery.

Next, the Iliac osteotomy is made. A reciprocating saw is used to divide the iliac blade down as far as the pelvic brim, where there is an obvious ridge. This osteotomy begins $2 \mathrm{~cm}$ above the anterior superior iliac spine and is directed straight posteriorly, allowing leeway above the joint. The saw blade is then re-orientated and turned through around $60^{\circ}$ in order to achieve an angle parallel to the original posterior column osteotomy. The saw blade is used to complete the osteotomy medially. This step is performed with relative ease because the original posterior column osteotomy is advanced so proximally early on in the operation. The final completion is then made in the same plane, joining the posterior column osteotomies with the Ganz osteotome (Fig. 7). This completes the peri-acetabular osteotomy.

Correction of the deformity is now possible, as the acetabular fragment is now free. A Schanz pin is placed under power into the acetabular fragment through the anterior inferior iliac spine, granting control of the fragment. The correction of orientation is confirmed under fluoroscopic guidance. At this stage, the degree of lateral and anterior femoral head coverage can be altered, dependent on the correction desired in the specific patient. To augment the correction, it is often necessary for an assistant to push the fragment, displacing and translating it laterally (Fig. 8). Images necessary at this point include a true antero-posterior projection, which allows assessment of the lateral cover and the Tönnis angle. A false profile projection will allow appreciation of the anterior cover and anterior centre-edge angle (Fig. 9). 
Fig. 3 Illustration of the same projection. The medial and lateral Hohmann retractors are seen around the ischium, with the lateral retractor protecting the sciatic nerve. Furthermore, the approach allows a much more advantageous position for the surgeon to place the osteotome in the direction of the osteotomy. The obturator nerve is seen as it appears in this medial approach
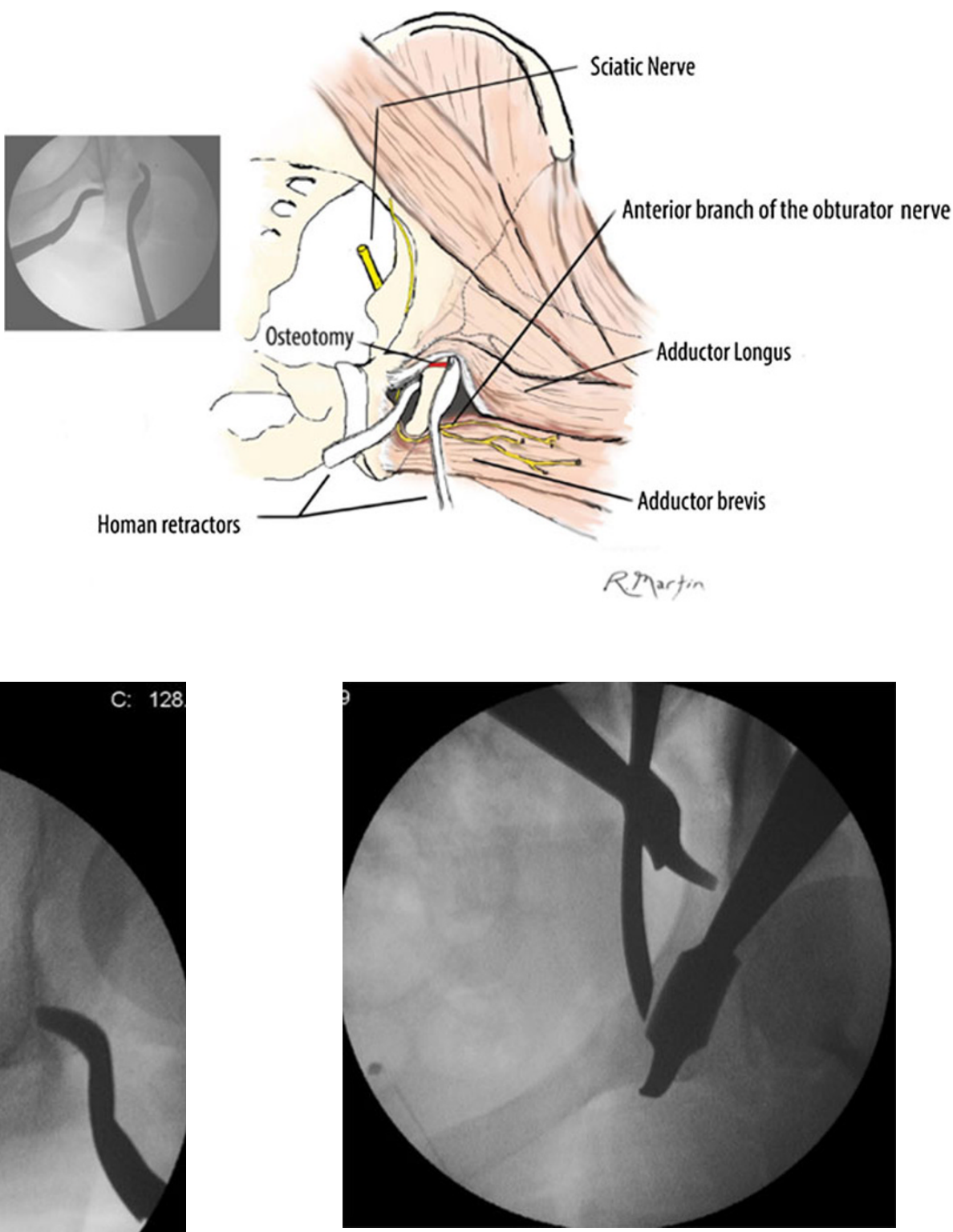

Fig. 5 Curved osteotome to the pubis following release of the periosteum. Retractors are for visualisation and protection of the soft tissues

extension of the iliac incision, inferior to the anterior superior iliac spine, is avoided altogether, with only a very small, more proximal scar being left. This seems to be far better tolerated from a cosmetic point of view. The medial scar is not only very small and heals well, but is entirely forgotten about by patients. The more extensive dissection distally over the anterior hip capsule as described in the classical Ganz peri-acetabular osteotomy is also avoided. The orientation of the pubic osteotomy described is also felt to augment the multi-planar correction.

It is outside of the scope of this technical description to detail the results achieved; however, we have performed in 


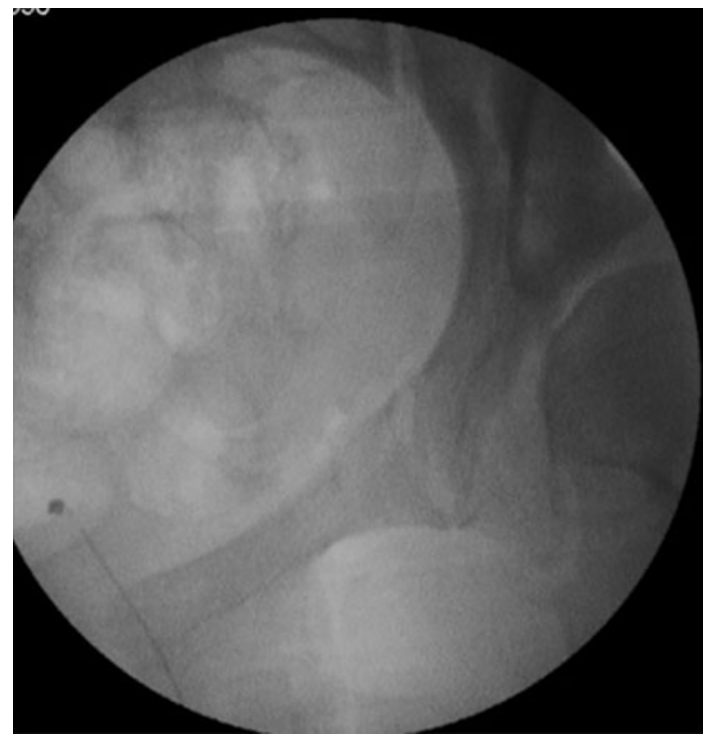

Fig. 6 Curved osteotomy of the pubis, medial to the pubic eminence but close to the joint

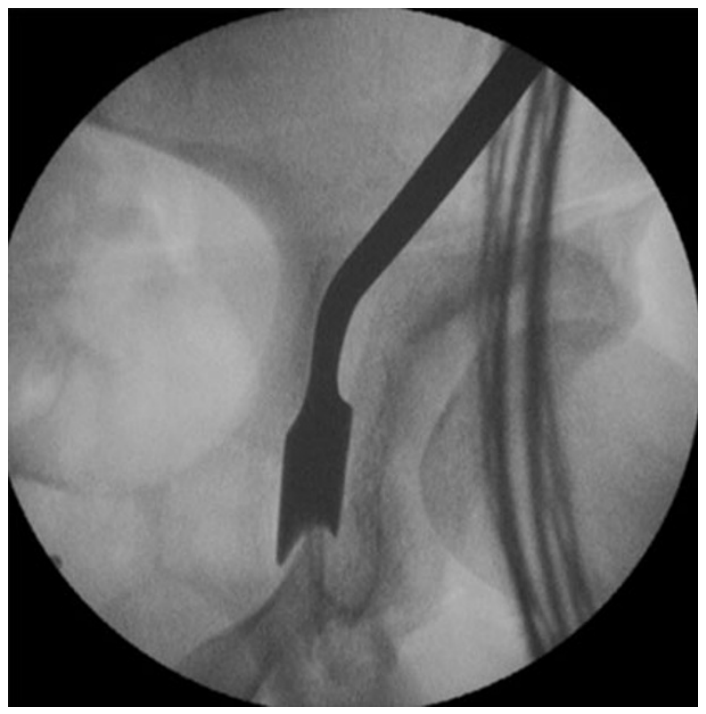

Fig. 7 Joining the posterior column osteotomies with the Ganz osteotome

excess of 90 peri-acetabular osteotomies via this approach since 2003 and many more triple osteotomies prior to this. Complications have been limited to one delayed union of the ischium (union at 9 months), one delayed union of the ilium, which healed after bone grafting, and the same patient suffered a non-fatal pulmonary embolus. There has also been one sciatic neurapraxia, which recovered after $48 \mathrm{~h}$ once we discontinued a sling device behind the thigh that had been used for patient comfort.

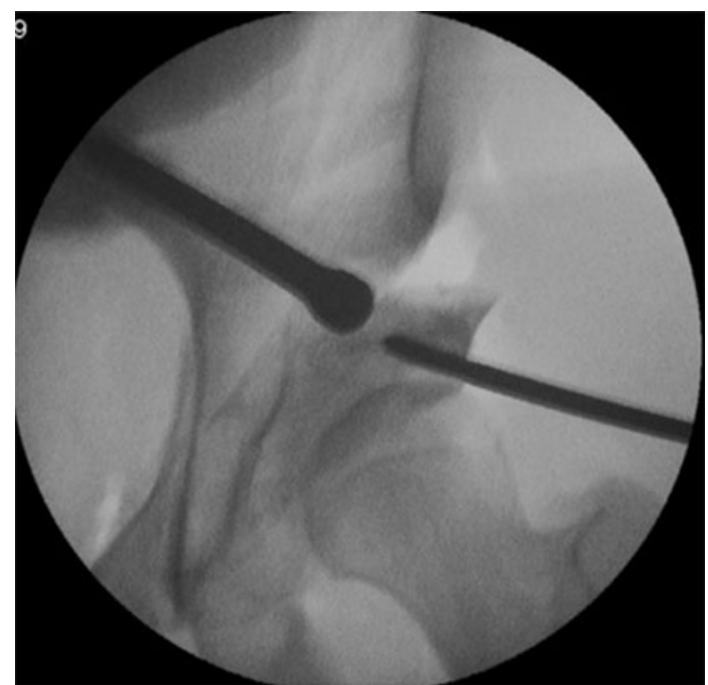

Fig. 8 Achieving correction with a Schanz pin in the mobile fragment and pusher medially to help gain lateral coverage

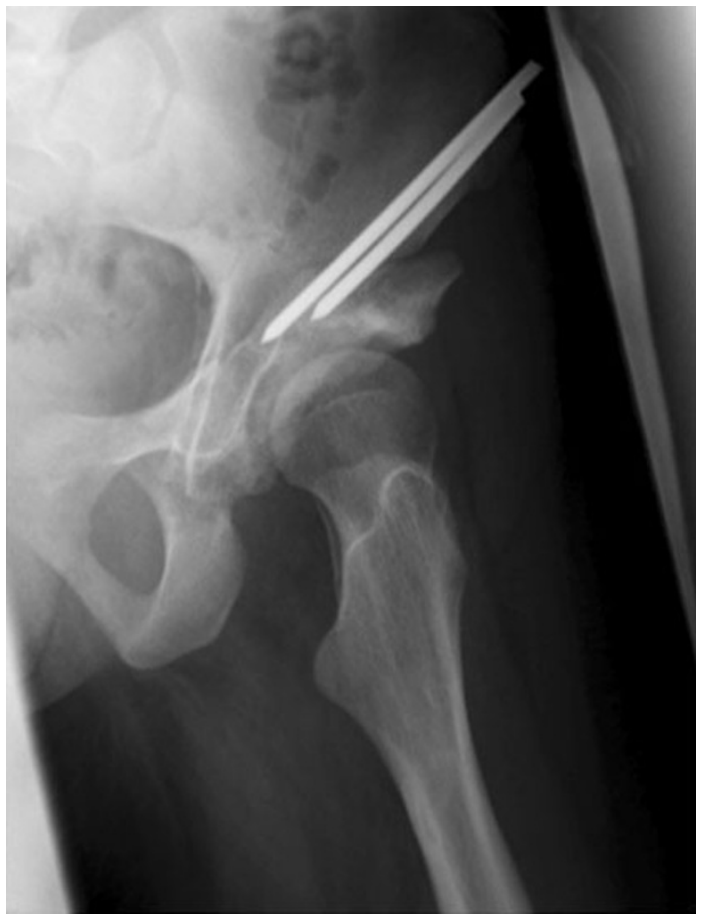

Fig. 9 False profile projection, allowing appreciation of the correction and visualisation of the anterior centre-edge angle

Acknowledgements The authors would like to thank Dr. Ryan Martin for his work on the illustrations in this paper.

\section{Reference}

1. Ganz R, Klaue K, Vinh TS, Mast JW (1988) A new periacetabular osteotomy for the treatment of hip dysplasias. Technique and preliminary results. Clin Orthop Relat Res 232:26-36 